\title{
The response of the azimuthal component of the ionospheric electric field to auroral arc brightening
}

\author{
V. Safargaleev ${ }^{1}$, W. Lyatsky ${ }^{1}$, N. G. Gazey ${ }^{2}$, P. N. Smith ${ }^{3}$, V. Kriviliov ${ }^{1}$ \\ ${ }^{1}$ Polar Geophysical Institute, Apatity, 184200, Russia \\ E-mail: safar@pgi.kolasc.net.ru \\ ${ }^{2}$ EISCAT group, Rutherford Appleton Laboratory, Chilton, Oxfordshire, OX11 0QX, UK \\ ${ }^{3}$ Space Physics Group, University of Sussex, Brighton, E Sussex BN1 9QH UK
}

Received: 26 October 1998 / Revised: 11 June 1999 / Accepted: 30 June 1999

\begin{abstract}
We have analyzed the response of azimuthal component of the ionospheric electric field to auroral arc activity. We have chosen for analysis three intervals of coordinated EISCAT and TV observations on 18 February, 1993. These intervals include three kinds of arc activity: the appearance of a new auroral arc, the gradual brightening of the existing arc and variations of the arc luminosity. The arcs were mostly east-west aligned. In all cases, the enhancement of arc luminosity is accompanied by a decrease in the westward component of the ionospheric electric field. In contrast, an increase of that component seems to be connected with arc fading. The observed response is assumed to have the same nature as the "short circuit" of an external electric field by the conductor. The possible consequence of this phenomenon is discussed.
\end{abstract}

Key words. Ionosphere (electric fields and currents; ionospheric irregularities) $\cdot$ Magnetospheric physics (auroral phenomena)

\section{Introduction}

The horizontal electric fields near and within auroral arcs have been extensively studied for more than two decades using radars, rockets, and satellites. Two arc classification schemes have been proposed, based on observations of electric fields by de la Beaujardiere et al. (1981) and Marklund (1984). The large-scale pattern of electric fields near arcs, identified only as a region of enhanced electron density, was presented by Opgenoorth et al. (1990) and confirmed later by Williams et al. (1992); Aikio et al. (1993); Lewis et al. (1994) and others. Large electric fields are mostly found on the

Correspondence to: V. Safargaleev equatorward side of an arc in the pre-midnight sector and on the poleward side after local midnight, are seen as enhancements of the background convection electric field and are directed toward the arc.

The studies mentioned focused on the variation of the normal (meridional) component of the field through the arc. However, the investigation of the tangential (azimuthal) electric field is also important for understanding the arc electrodynamics because of the possible role of this component in the arc generation (see Borovsky, 1993 for references). Nevertheless, relatively little attention is still paid to this study. One case was mentioned by Kirkwood et al. (1988), and a number of papers concentrated on this component in connection with substorm investigation (Kozelova et al., 1982; Kirkwood et al., 1988; Lewis et al., 1997). Haerendel et al. (1993) and Gazey et al. (1996) discussed azimuthal electric fields in connection with studies of the motion of auroral arcs relative to plasma convection in the ionosphere.

Another problem is to investigate the temporal variations of the electric field. Aikio et al. (1993) reported the simultaneous intensification of the arcassociated normal electric field with the optical brightening of the arc. A similar case had been mentioned before by Timofeev et al. (1987). In contrast, Lewis et al. (1994) concluded that enhanced arc-associated electric fields are connected spatially to the arcs and move with them.

The use of high-resolution TV-data is one of the ways to resolve the problem. It is interesting to note a result by Lanchester et al. (1996). They compared high time resolution optical measurements (TV camera and filter photometer) with horizontal electric field measurements and found the electric field to point towards the bright optical features which were moving along the arc.

In this study we compare the variations of the azimuthal component of ionospheric electric field with variations of auroral arc luminosity. The arcs were observed by TV-camera, the incoherent EISCAT radar provided the measurements of the ionospheric electric fields. 


\section{Description of measurements}

The measurements were made during an observing campaign near Tromsø, Norway on 18 February, 1993. The plasma convection velocities and the corresponding electric fields were measured with the tristatic UHF EISCAT radar. For a detailed description of the EISCAT radar see Folkestadt et al. (1983). The transmitter station is located at Ramfjordmoen $\left(69.59^{\circ} \mathrm{N}\right.$ and $19.23^{\circ} \mathrm{E}$ ) near Tromsø. The other two passive stations at Kiruna $\left(67.86^{\circ} \mathrm{N}\right.$ and $\left.20.44^{\circ} \mathrm{E}\right)$ and Sodankyla $\left(67.37^{\circ} \mathrm{N}\right.$ and $26.65^{\circ} \mathrm{E}$ ) make measurements at the single intersection point of the three antenna beams at the altitude of $348 \mathrm{~km}$. Eastward component of horizontal electric field (30-s integration) was used for analysis.

The TV camera was situated at the Tromsø transmitter station. It was pointed towards the geographic zenith, and the fish-eye lens gave a circular field of view of $180^{\circ}$. In Fig. 1a the location of the TV camera and the EISCAT transmitter is shown by a black circle, the projection of the single intersection point $\left(68.93^{\circ} \mathrm{N}\right.$ and $19.20^{\circ} \mathrm{E}$ ) is shown by a black square, and the TV camera field of view is bordered by the large circle for a height of $100 \mathrm{~km}$.

The TV data for the period of interest were digitised at $1 \mathrm{~s}$ intervals with spatial resolution of about $0.6 \times 0.6 \mathrm{~km}$ at an altitude of $110 \mathrm{~km}$ near the TV camera zenith. The examples of the digitised TV frames are presented in Fig. 1b. They show the location of the auroras relative to the EISCAT zenith for the events under consideration. Also shown are (Z)enith, (E)astward and (W)estward splits which are used to construct the keograms and calculate the mean value of the arc luminosity as well as the luminosity of the sky near the EISCAT zenith. The width of the Z-split is about $30 \mathrm{~km}$ at an altitude of $110 \mathrm{~km}$ near the zenith.

The ground magnetic field was measured in Soroya (SOR) $\left(70.54^{\circ} \mathrm{N}\right.$ and $\left.22.22^{\circ} \mathrm{E}\right)$, Kilpisjarvi (KIL) $\left(69.02^{\circ} \mathrm{N}\right.$ and $\left.20.79^{\circ} \mathrm{E}\right)$, Muonio (MUO) $\left(68.02^{\circ} \mathrm{N}\right.$ and $23.53^{\circ} \mathrm{E}$ ) and Sodankyla (SOD) geomagnetic observatories at 10,10, 10 and 15 -s resolution, respectively. The SOR, KIL and MUO observatories are the part of the IMAGE network.

\section{Results of observation}

\subsection{Overview}

The data were taken during a magnetically active period which lasted for many days. The day of February 18th was characterized by a mean value of $K_{p} \approx 4$. Magnetic data show at least four onset-type events during the interval between 1800-2400 UT. The IMF's Z-component was directed southward for several hours before and during these events, the auroral activity was also very high (Gazey et al., 1996).

The $\mathrm{X}$ and $\mathrm{Z}$ component magnetic records from the auroral region in Scandinavia are illustrated in Fig. 2a, b respectively. The aurora enhancement times are marked with vertical lines. Two events of interest (2029 UT and

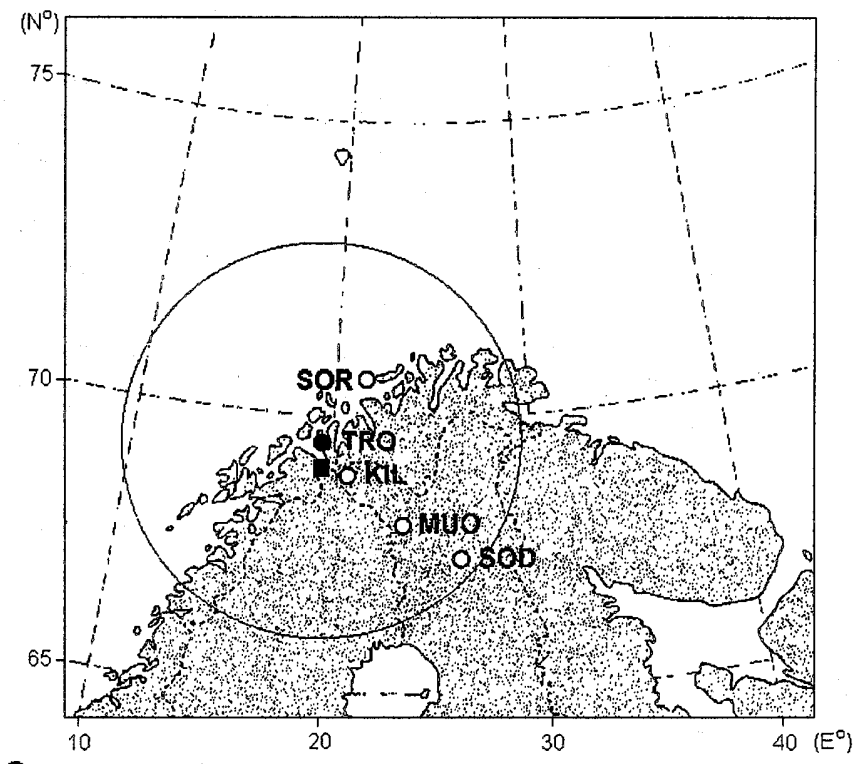

a

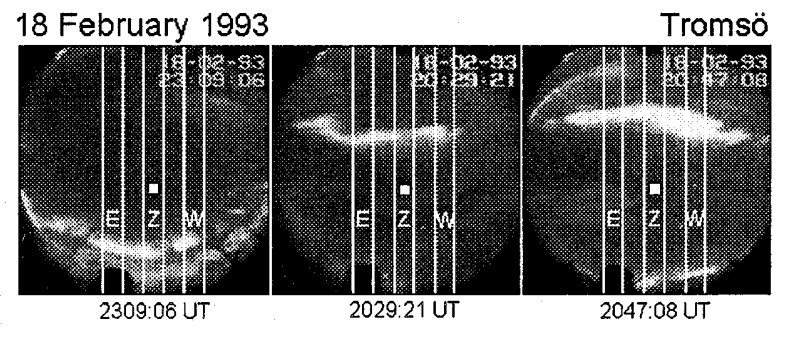

Fig. 1. a Map showing the location of the EISCAT transmitter and TV-camera (black circle), as well as magnetic stations (open circles); the large circle indicates the field of view of the TV-camera, the black square shows the EISCAT zenith at an auroral height of $100 \mathrm{~km}$. b TV-images showing the co-location of the auroral arcs and the EISCAT zenith marked with white squares

2047 UT) occur a few minutes before substorm onset started near 2048 UT as a negative bay in the X component in KIL, MUO and SOD (Fig. 2a) and accompanied by Pi2 pulsations in SOD (not shown). During this time Scandinavia was situated in the premidnight sector (local magnetic midnight is at $2130 \mathrm{UT}$ ). The $\mathrm{Z}$ component magnetic fields (Fig. 2b) reveal that a substorm-associated westward electrojet lay south of the Tromsø site, somewhere between KIL and MUO. The event at 2309 UT was also accompanied by rather weak enhancement in this electrojet. The $\mathrm{Z}$ component determinations of the latitude of the centre of the electrojet are confirmed by the location of the maxima in the disturbance in the $\mathrm{X}$ components.

\subsection{Appearance of a single auroral arc at 2309 UT (event 1)}

We start from the interval which is the simplest to analyze. The auroral activity for this interval is presented in Figs. 1b, 3a and 4a (top panel). It is characterized by the appearance of a new auroral arc and fading of pre-existing arc at the southern edge of Tromsø 

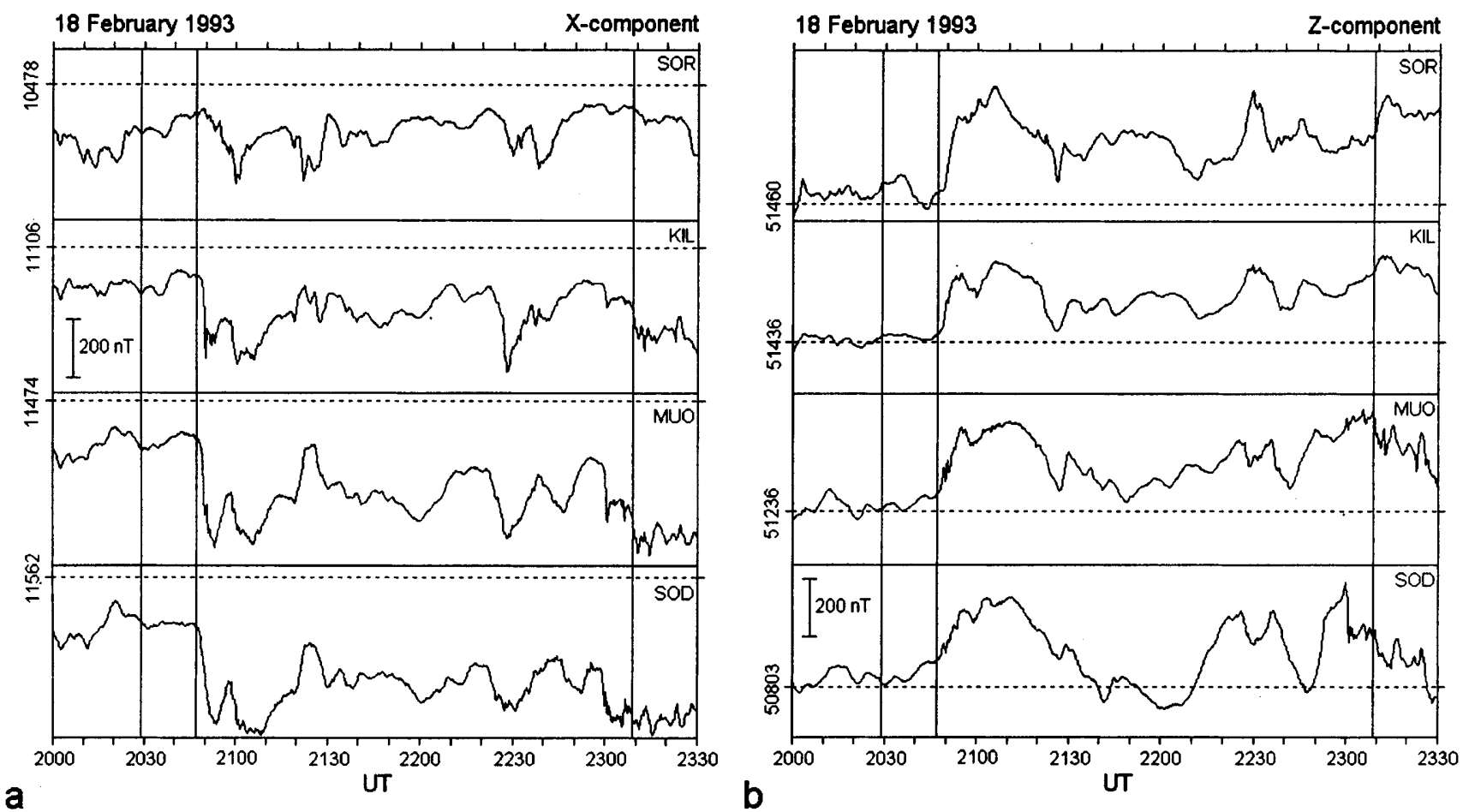

Fig. 2a, b. High-latitude magnetograms from the Scandinavia during the intervals studied: a X components, $\mathbf{b} \mathrm{Z}$ components. The times of the aurora arc enhancement are marked with vertical solid lines

TV-camera field of view. Also shown are the variations of the eastward component of the ionospheric electric field (black step-like traces in Figs. 3 and 4) measured by EISCAT radar approximately $12^{\circ}$ south of the zenith.

The dynamics of aurora equatorward the EISCAT beam is presented on the keogram in Fig. 3a, (in this the trace of the EISCAT zenith at the auroral altitude coincides with the upper border). This keogram (as well as another two keograms below) is obtained along the Z-profile (see Fig. 1b). Near 2309 UT, a new arc appears in the ionosphere approximately $200 \mathrm{~km}$ equatorward the EISCAT beam. Its appearance is accompanied by a distinct decrease in westward component of the ionospheric electric field from $15 \mathrm{mV} / \mathrm{m}$ to $2 \mathrm{mV} / \mathrm{m}$ near the EISCAT zenith. The variations of the arc luminosity are shown in Fig. 4a (top) where the appearance of a new arc is seen both on Z-profile and W- and E-profiles almost simultaneously. For comparison we present also the variations of sky luminosity near the EISCAT zenith (Fig. 4a, bottom). There are no significant changes during the period.

As seen in Figs. 1b, 3a, the appearance of new arc is accompanied by a fading of pre-existing one. The similar variations of the luminosity in the system of two parallel arcs were reported early by Safargaleev et al. (1997).

\subsection{Increase of the luminosity of a single auroral arc at 2029 UT (event 2)}

In this section we examine the response of the azimuthal electric field to the gradual increase in the luminosity of single auroral arc drifting slowly toward the Tromsø local zenith. The dynamics of the arc during the interval is shown on the keogram in Fig. 3b, the luminosity of the arc versus time is presented in Fig. 4b (top panel), (in Fig. 3b, $c$ the trace of the EISCAT zenith at the auroral altitude coincides with the lower borders). The gradual increase in the luminosity started at 2028:30 UT and was seen both on Z-profile and W- and E-profiles. The arc was at a distance of about $100 \mathrm{~km}$ from the EISCAT beam when the arc luminosity reached the maximum. At this time (near 2029 UT) the decrease in the westward component of ionospheric electric field from $17 \mathrm{mV} / \mathrm{m}$ to $2 \mathrm{mV} / \mathrm{m}$ is detected by EISCAT. After that the arc begins to fade which accompanied by small increase in the electric field.

As for the previous case, we have examined also the luminosity of the sky (shown by curves in Fig. 4b, bottom panel) as well as variations of the mean value of the electron concentration in the ionospheric E-region (dashed step-like traces in the same panel) near the EISCAT beam. No significant changes both in luminosity and density were seen again through the interval.

\subsection{Luminosity variations in a single auroral arc around 2047 UT (event 3)}

The period includes the substorm expansion phase which starts as a negative excursion in the X-component at 2048:15 UT (see Fig. 2a). The auroral breakup begins as a brightening of the arc near southward edge of TV-camera field of view. Approximately ten minutes later, the westward travelling surge appears east of Tromsø. The onset was preceded by a period of 

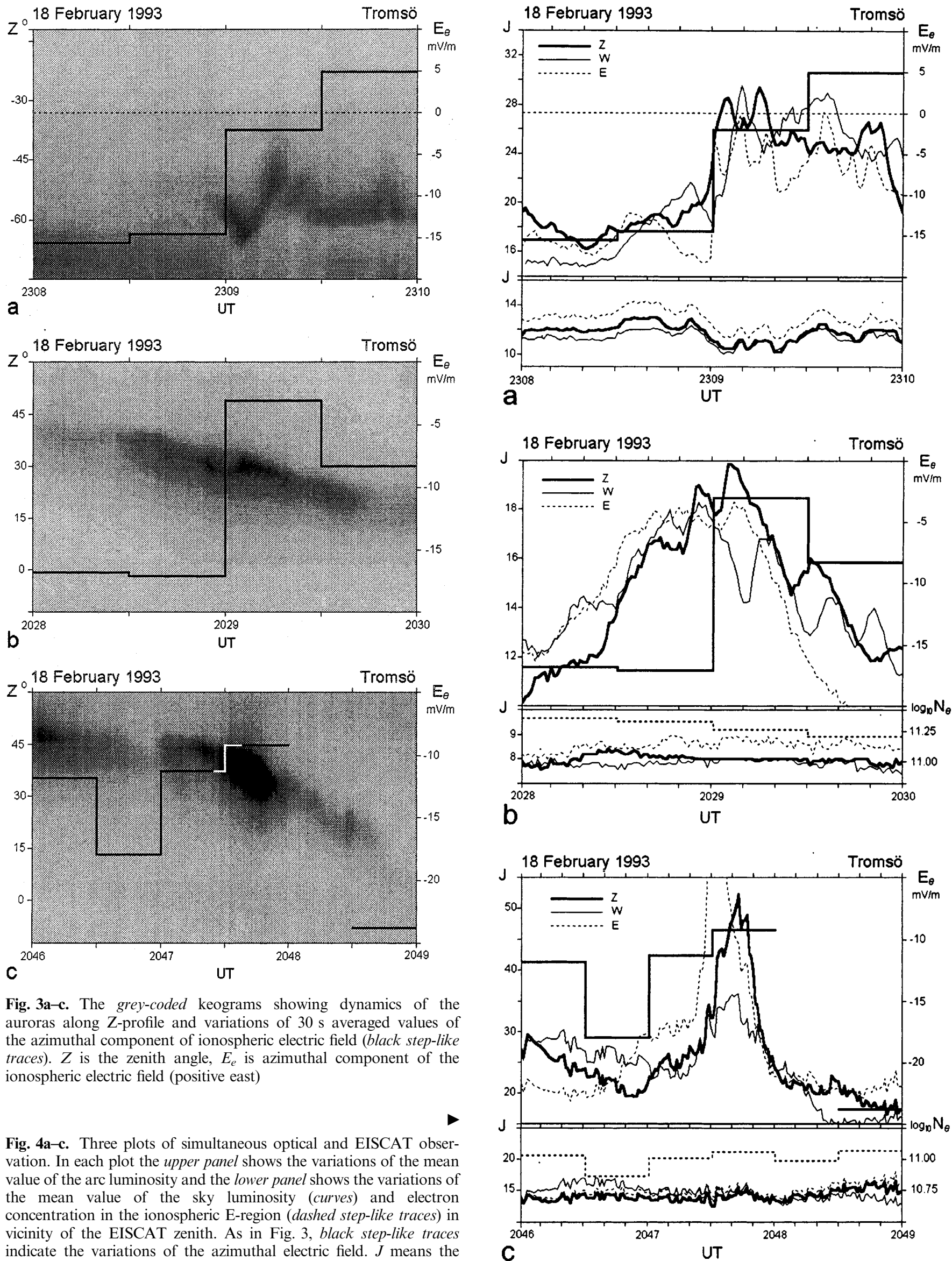

Fig. 3a-c. The grey-coded keograms showing dynamics of the auroras along Z-profile and variations of $30 \mathrm{~s}$ averaged values of the azimuthal component of ionospheric electric field (black step-like traces). $Z$ is the zenith angle, $E_{e}$ is azimuthal component of the ionospheric electric field (positive east)

Fig. 4a-c. Three plots of simultaneous optical and EISCAT observation. In each plot the upper panel shows the variations of the mean value of the arc luminosity and the lower panel shows the variations of the mean value of the sky luminosity (curves) and electron concentration in the ionospheric E-region (dashed step-like traces) in vicinity of the EISCAT zenith. As in Fig. 3, black step-like traces indicate the variations of the azimuthal electric field. $J$ means the intensity of the luminosity (arbitrary units) 
relatively low magnetic activity. During this period the TV-camera showed two auroral arcs, a very weak arc near the local zenith and rather intense arc poleward of them. At 2046 UT the weakest arc disappears almost completely.

An interval under consideration starts at 2046 UT when the arc near EISCAT beam has faded and includes the quasi-periodical variation of the activity in single arc located approximately $100 \mathrm{~km}$ north of zenith. The dynamics of the arc is shown on the keogram in Fig. 3c, the luminosity of the arc versus time is presented in Fig. 4c (top panel). The black step-like trace shows the variations of the azimuthal component of ionospheric electric field.

Three steps of auroral development can be seen in Figs. 3c and 4c. At first the arc fades. At 2047 UT the arc luminosity starts to increase and reaches a maximum near 2047:40 UT. After that the arc fades again and disappears. During the period the arc drifts equatorwards. The character of the arc dynamics seems to be typical for pseudobreakup events (Koskinen et al., 1993).

The increase of the arc activity after 2047 UT is accompanied by a decrease in the westward component of background electric field from $18 \mathrm{mV} / \mathrm{m}$ to $8 \mathrm{mV} / \mathrm{m}$. In contrast, disappearance of the arc near 2048:30 UT and fading near 2046:30 UT are accompanied by increases in this component from $10 \mathrm{mV} / \mathrm{m}$ to $25 \mathrm{mV} / \mathrm{m}$ and from $7 \mathrm{mV} / \mathrm{m}$ to $15 \mathrm{mV} / \mathrm{m}$, respectively. As for the previous cases, neither sky luminosity nor electron concentration varies significant near radar beam during the period (see bottom panel in Fig. 4c).

\section{Discussion}

\subsection{Polarization electric fields generated by an elliptic ionospheric inhomogeneity}

In the present study we have analyzed the variations of the azimuthal component of ionospheric electric field and of aurora luminosity. The observed correlation of these variations allows us to conclude that a decrease in the westward component of the field is caused by increase of auroral arc luminosity, whereas the increase in this component seems to be connected with the arc fading. The optical observations near the measurement point also support our assumption as they do not show any significant variations of the sky luminosity here.

We think that the local enhancement of ionospheric conductivity may be the most probable reason of the phenomenon observed. If the magnetospheric generator region acts as a current generator, the ionospheric electric field has to modify itself in a way that the current continuity in the ionosphere is preserved. From this point of view Aikio et al. (1993) discussed the increase of the meridional electric field in the adjacent arc region of low ionospheric conductivity. In our study, the ambient electric field has a significant azimuthal component, and the appearance of the azimuthally confined region of enhanced conductivity causes this component to decrease. Qualitatively this effect is similar to the "short circuit" of an external electric field by a conductor. Quantitatively it may be described in terms of a polarization electric field that arises inside and around the stretched ionospheric inhomogeneity and results in a decrease the ambient field.

The disturbed electric fields generated by a circular region of enhanced conductivity had been calculated by Maltsev et al. (1974). For an elliptic inhomogeneity, the expressions for the electric disturbances may be inferred from Eqs. $(13,15)$ taken from Maltsev et al. (1974) by the conformal transformation $z=\varsigma+(a+b) / 4 \varsigma$ of an ellipse on the complex plane $z$ into a circle ring on the complex plane $\varsigma$. (Here $a$ and $b$ are the major and minor semi-axes of the ellipse). For the model in Fig. 5 (top panel) they have the following form (see also Lyatsky and Maltsev, 1983):

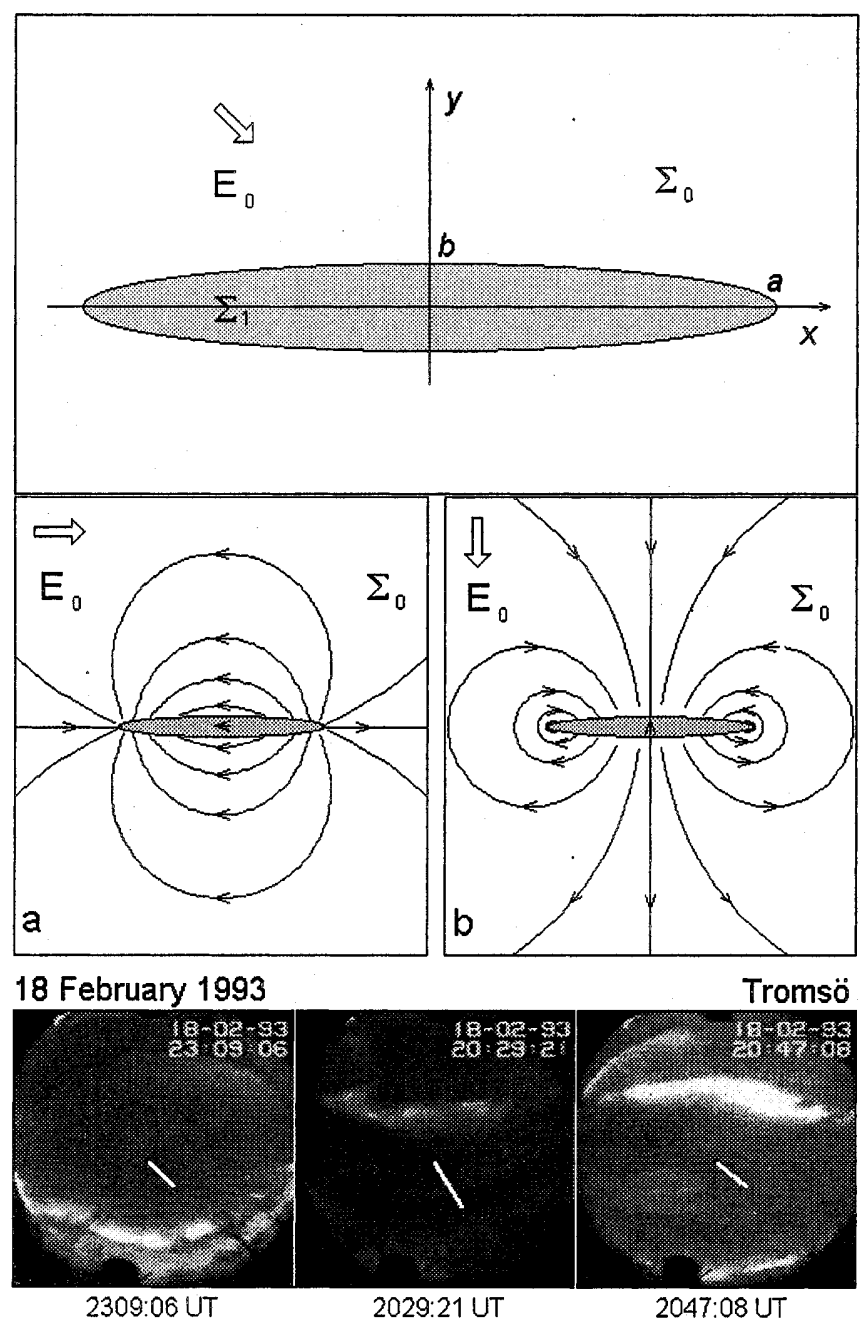

Fig. 5a, b. Elliptic inhomogeneity in the ionosphere; $E_{0}$ and $\Sigma_{0}$ are undisturbed background electric field and conductivity, respectively, and $\Sigma_{1}$ refers to the region of enhanced conductivity (top panel). The distribution of polarization electric fields generated by the ionospheric inhomogeneity orientated along (central panel, a) and across (central panel, b) the ionospheric electric field. The images showing the orientation of the arcs under consideration relatively the ionospheric electric field (bottom panel) 
$E_{1}=\frac{a+b}{a-b} \frac{\Sigma_{0}+\Sigma_{0}^{*}+2 \Sigma_{w}}{\Sigma_{1}-\Sigma_{0}} \frac{\mu E_{0}+E_{0}^{*}}{\mu \mu^{*}-1}-E_{0}$

where

$\mu=\frac{a+b}{a-b} \frac{\Sigma_{1}^{*}+\Sigma_{0}+2 \Sigma_{w}}{\Sigma_{1}^{*}-\Sigma_{0}^{*}}$

$E_{2}=-\frac{1}{2}\left(E_{1}+\frac{a+b}{a-b} E_{1}^{*}\right)\left(\frac{1}{\sqrt{1-\frac{a^{2}-b^{2}}{z^{2}}}}-1\right)^{*}$

Equation (1) gives the value of the disturbed electric field, $E_{1}$, inside the ellipse, and Eq. (2) describes the disturbance of electric field outside the inhomogeneity. For convenience all vectors were replaced by complex numbers of the kind $E=E_{x}+i E_{y} . z=x+i y$ is the complex independent variable (distance). $\Sigma=\Sigma_{\mathrm{P}}-i \Sigma_{\mathrm{H}}$ is the complex conductivity and $\Sigma_{\mathrm{P}}$ and $\Sigma_{\mathrm{H}}$ are the heightintegrated Pedersen and Hall conductivities of the ionosphere (indexes 0 and 1 correspond to the background and disturbed values, respectively); $\Sigma_{w}$ is the effective conductivity of the magnetospheric plasma for propagating Alfvén wave, $\Sigma_{w}=\left(\mu_{0} V_{A}\right)^{-1}$, where $\mu_{0}$ is the vacuum magnetic permeability, $V_{A}$ is the Alfven velocity. The symbol '*' means the complex conjugate. The Eqs. (1-2) are obtained by assuming that charges and field aligned currents are located on the ellipse periphery, and the undisturbed electric field $\mathbf{E}_{0}$ is uniform.

The distribution of the polarization electric field around the inhomogeneity is presented in Fig. 5 (central panel) for two cases of orientation of the inhomogeneity in the background electric field. For the idealized model of global convection (see, i.e. Kan and Sun, 1996) case $a$ corresponds to the arc which appears in the auroral ionosphere near local midnight at the preliminary phase of a substorm when the magnetospheric convection is forced and ionospheric electric field is mostly E-W oriented. Case $b$ corresponds to the arc placed in the morning or evening sector of auroral zone where the convection electric fields are meridional electric fields. In reality, the distribution of the ionospheric electric field near local midnight is rather complex. For the events under consideration the angle between the arc and electric field was about $45^{\circ}$ (see Fig. 5, bottom).

For the inhomogeneity stretched along the field (Fig. 5a), there is a large area where the disturbed electric field is directed against the ambient field. So, the tangential component of the total electric field will decrease here. In the case of perpendicular orientation (Fig. 5b), the response of the tangential component to the appearance of the inhomogeneity will strongly depend on the location of the measurement point.

We present the solution of Eqs. (1-2) for the polarization electric fields generated by an elliptic inhomogeneity stretched along the ambient electric field. To estimate the disturbance of ionospheric conductivity inside the inhomogeneity, we have calculated the highintegrated Pedersen and Hall conductivity using the real profiles of electron density measured by EISCAT and the values of neutral density and undisturbed electron temperature taken from the CIRA (1972) model. The results of calculations are presented in Fig. 6. Note, that during the intervals of interest (marked with black arrows in Fig. 6) the ionospheric conductivity near EISCAT beam almost did not change, whereas inside the active region crossing the EISCAT beam near 2050 UT the conductivity increases significantly.

The solution of Eqs. (1-2) for the tangential component of disturbed electric field outside the inhomogeneity, $E_{2}$, is presented in Fig. 7 for different values of parameters. In particular, assuming $\Sigma_{\mathrm{H}} / \Sigma_{\mathrm{P}}=4, \Sigma_{\mathrm{H} 0} /$ $\Sigma_{\mathrm{P} 0}=2$, for the inhomogeneity of $20 \times 1000 \mathrm{~km}$ and $\Sigma_{\mathrm{P}} / \Sigma_{\mathrm{P} 0} \sim 10$ we have $E_{2} \cong-0.5 E_{0}$ at a distance of $100 \mathrm{~km}$ from the inhomogeneity. For the $45^{\circ}$ orientation, the disturbed field at $100 \mathrm{~km}$ has approximately the same value near the $y$-axis (at $x \sim 0$ ), but is three times smaller near the inhomogeneity edges (at large $|x|$ ). For the events under consideration we get the disturbance from $-0.4 E_{0}$ to $-0.8 E_{0}$.

It is necessary to note that our model does not take into account the possible influence of the magnetosphere where the polarization electric fields can, for example, partially close via field-aligned currents. Most of the self-consistent models proposed assume the arc to be a narrow strip, infinitive or homogeneous in the east-west direction (Borovsky, 1993; Kozlovsky and Lyatsky, 1994). The task becomes rather complex if the ionospheric irregularity is also azimuthally confined. We regard this question as beyond the scope of this study and emphasis.

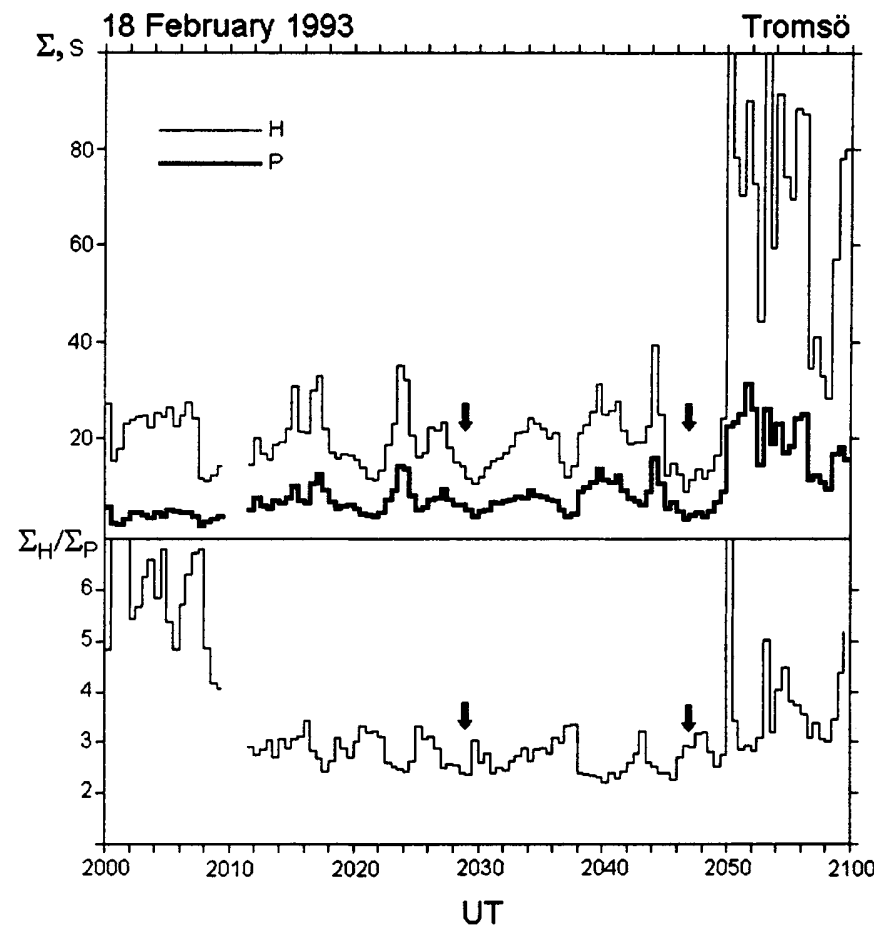

Fig. 6. Temporal variations of the high-integrated Hall and Pedersen conductivity (top panel) and the ratio of Hall to Pedersen conductivity (bottom panel) measured by EISCAT; black arrows indicate the moments under consideration 

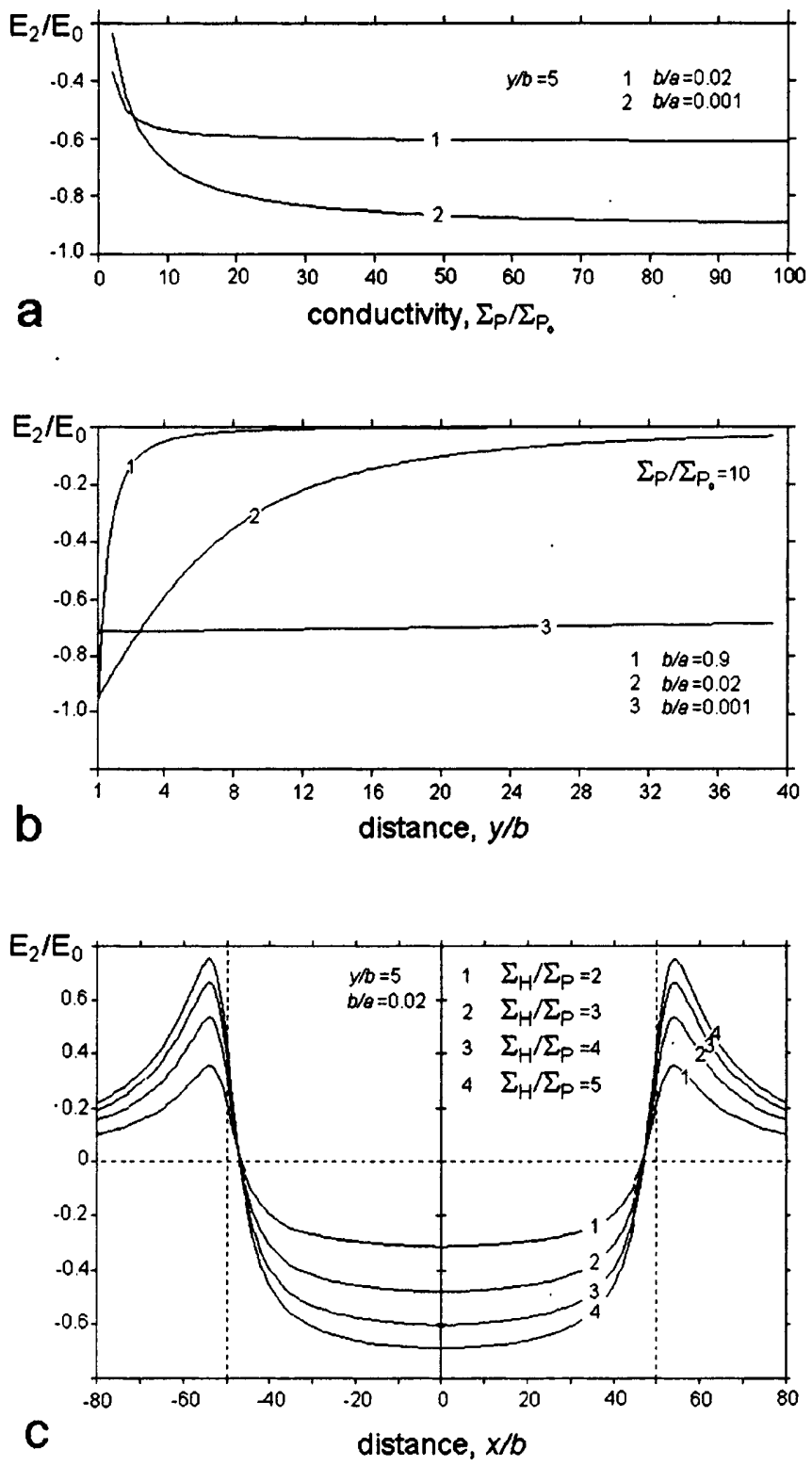

Fig. 7a-c. The distribution of the azimuthal component of polarization electric field, $E_{2}$, generated by the ionospheric inhomogeneity stretched along the ambient electric field. $\mathbf{a}$ is the electric field outside the inhomogeneity versus the conductivity disturbance and $\mathbf{b}$ the distance from inhomogeneity, and $\mathbf{c}$ the distribution of the electric field along the inhomogeneity. $\Sigma_{\mathrm{p} 0}$ is the height-integrated Pedersen conductivity, $\Sigma_{\mathrm{p}}$ is the disturbance of Pedersen conductivity inside the inhomogeneity

\subsection{Out-of-phase variation of the luminosity in the system of two parallel arcs}

Recently Safargaleev et al. (1997) have reported the "out-of-phase" variations of the arc intensity when the appearance of a new arc was accompanied by fading or disappearance of a pre-existing one. They proposed the decrease of the azimuthal component of ionospheric electric field as one of the possible reason "switching off" the pre-existing arc. For instance, Borowsky (1993) discussed a model in which the width of the arc is

proportional to the magnitude of tangential component of the background electric field.

As was mentioned in Sect. 3.1, we observed the "outof-phase" event during event 1 (see Figs. 1b, 3a). In Fig. 8 we present the variations of the mean value of luminosity in the equatorward (pre-existing) and poleward (appearing) arcs. It is clear that the appearance of a new arc is accompanied by a decrease in the westward component of ionospheric electric field and by the fading of pre-existing arc. Thus, the hypothesis by Safargaleev et al. (1977) is in an agreement with our observation.

\subsection{Behaviour of the meridional component of the electric field}

Variations of the meridional (normal) component of ionospheric electric field during the intervals under consideration are presented in Fig. 9. The black arrows indicate the moment of the azimuthal component reduction connected with appearance of new arc (Fig. 9a), maximum intensity in the brightening arc (Fig. 9b) and enhancement of the pre-breakup arc (Fig. 9c). In contrast to azimuthal component, no noticeable response to aurora enhancement is seen in the meridional component. This is not consistent with the results by Aikio et al. (1993). We think that although

18 February 1993

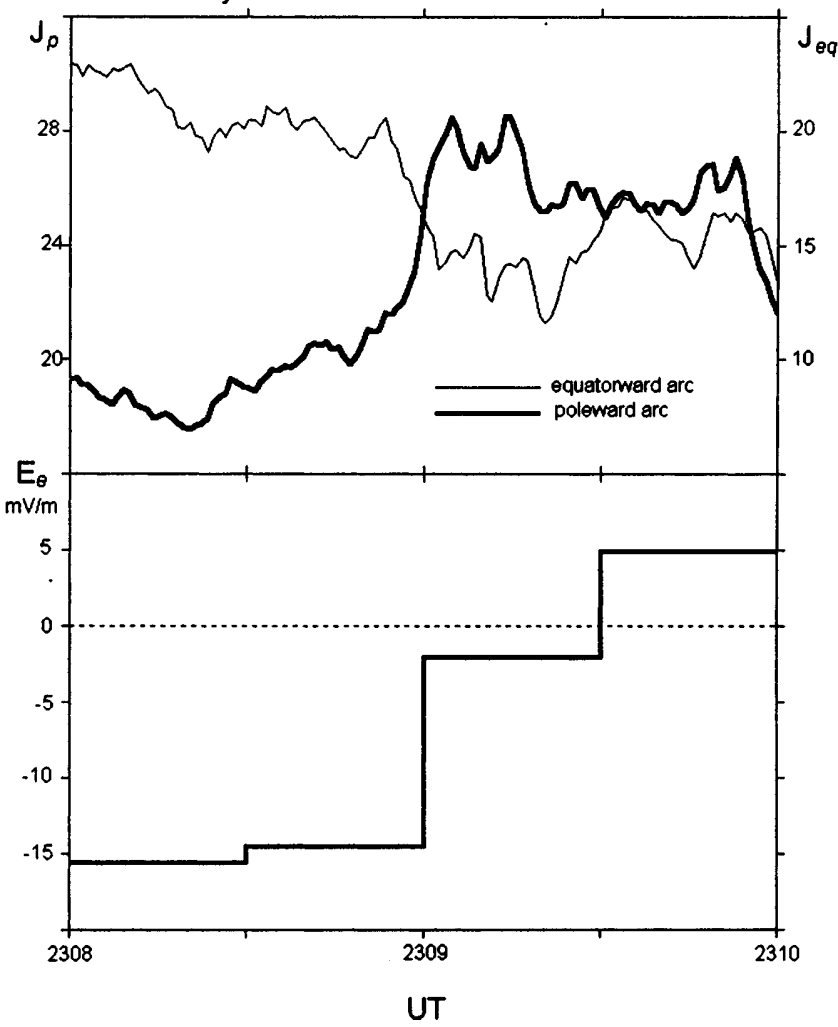

Fig. 8. Variations of the mean value of the luminosity in the system of two parallel auroral arcs (top panel) and variation of the azimuthal component of electric field in the ionosphere (bottom panel). $J$ means the intensity of the luminosity (arbitrary units) 

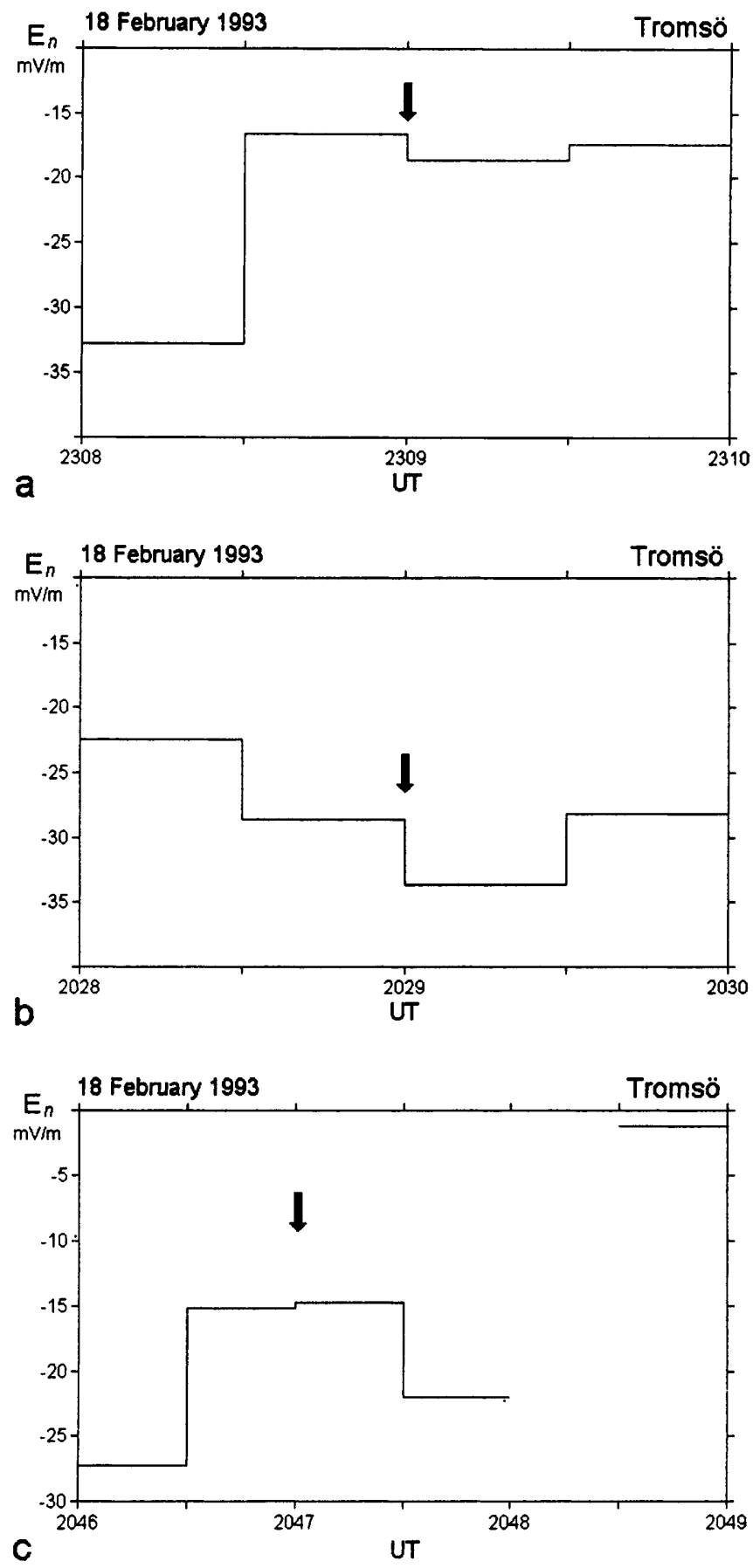

Fig. 9a-c. Variations of the meridional component of ionospheric electric field through the intervals under consideration. Black arrows indicate the times of azimuthal component decrease

the arc-associated polarization electric field changes this component of the background convection field, the small electric field generated in response to the fieldaligned current system may compensate for these changes.

Nevertheless, it is interesting to note for events 2 and 3 that the meridional component increases while the arc verges toward the EISCAT beam. This is in agreement with the assumption by Lewis et al. (1994) about the region of enhanced electric field equatorward the arc moving with the arc.

\section{Summary}

In the present study we have analyzed the response of the ionospheric electric field (azimuthal component) to auroral arc brightening. We have examined three types of auroral arc activity: appearance of a new arc, the gradual brightening of the existing arc and variations of the arc luminosity. It is found that the enhancement of arc luminosity is accompanied by a decrease in the westward component of the ionospheric electric field. In contrast, the increase in this component is connected with the arc fading. The observed response is described in terms of polarization electric fields, which are generated by an elliptic inhomogeneity in the ionosphere and reduce the tangential component of background convection electric field. We think that the decrease of the electric field may be a reason for out-of-phase variations of the auroral activity observed by Safargaleev et al. (1997) in the system of auroral arcs.

Acknowledgements. We thank the Director and staff of EISCAT for the use of their data in this work and the institutes who maintain the IMAGE magnetometer array. One of the authors (V.S) thanks Dr. I. V. McCrea for the additional EISCAT data, Dr Uula Kultima for the magnetic data from Sodankyla Geophysical Observatory (SGO), and is also grateful to Drs Esa Turunen (SGO), A. B. Pashin and Evgenya Belova (both at PGI) for their assistance as well as to Dr E. E. Timofeev (PGI) for his interest in this work. The work by Russian authors was supported by the Russian Foundation for Basic Researches grant 97-05-65894.

The Editor-in-chief thanks A. Aikio and G. Haerendel for their help in evaluating this paper.

\section{References}

Aikio, A. T., H. J. Opgenoorth, M. A. L. Persson, and K. Kaila, Ground based measurements of an arc-associated electric field, J. Atmos. Terr. Phys., 55, 797-807, 1993.

Borovsky, J. R., Auroral arc thickness as predicted by various theories, J. Geophys. Res., 98, 6101-6138, 1993.

Beaujardiere, O., R. de la Vondrak, R. Heelis, W. Hanson, and R. Hoffman, Auroral arc electrodynamic parameter measured by AE-C and Chatanika radar, J. Geophys. Res., 86, 4671, 1981.

Folkestadt, K., T. Hagfors, and S. Westerlund, EISCAT: an updated description of technical characteristics and operational capabilities, Radio Sci., 18, 867, 1983.

Gazey, N. G. J., P. N. Smith, R. P. Rijnbeek, M. Buchan, and M. Lockwood, The motion of auroral arcs within convective plasma flow, Proc. Third International Conference on Substorms (ICS-3), Versailles, Paris, May 1996, ESA, SP-389, 11-16, 1996.

Haerendel, G., S. Buchert, C. La Hoz, B. Raaf, and E. Rieger, On the proper motion of auroral arcs, J. Geophys. Res., 98, 60876099, 1993.

Kan, J. R., and W. San, Substorm expansion phase caused by an intense localized convection imposed on the ionosphere, J. Geophys. Res., 101, 27,271-27,281 1996.

Kirkwood, S., H. Opgenoorth, and J. S. Murphree, Ionospheric conductivities, electric fields and currents associated with auroral substorms measured by the EISCAT radar, Planet. Space Sci., 36, 1359-1380, 1988.

Koskinen, H. E. J., R. E. Lopez, R. J. Pellinen, T. I. Pulkkinen, D. N. Baker, and T. Bosinger, Pseudobreakup and substorm 
growth phase in the ionosphere and magnetosphere, J. Geophys. Res., 98, 5801-5813, 1993.

Kozelova, T. V., M. I. Pudovkin, I. A. Zhulin, and L. L. Lazutin, Electric fields during different phases of magnetospheric substorm as inferred from balloon measurements, Geomagn. Aeron. (in Russian), 22, 471-475, 1982.

Kozlovsky, A. E., and W. B. Lyatsky, Instability of magnetosphereionosphere convection and formation of auroral arcs, Ann. Geophysicae, 12, 636-641, 1994.

Lanchester, B. S., K. Kaila, and I. W. McCrea, Relationship between large horizontal electric fields and auroral arc elements, J. Geophys. Res., 101, 5076-5084, 1996.

Lewis, R. V., P. J. S. Williams, G. O. L. Jones, H. J. Opgenoorth, and M. A. L. Persson, The electrodynamics of drifting auroral arc, Ann. Geophysicae, 12, 478-489, 1994.

Lewis, R. V., M. P. Freeman, A. S. Rodger, G. D. Reevs, and D. K. Milling, The electric field response to the growth phase and expansion phase onset of a small isolated substorm, Ann. Geophysicae, 15, 289-299, 1997.

Lyatsky, W. B., and Y. P. Maltsev, Magnetosphere-ionosphere interaction (in Russian), Izdatel'stvo Nauka, Moscow, 1983.
Maltsev, Y. P., S. V. Leontyev, and W. B. Lyatsky, Pi-2 pulsations as a result of evolution of an Alfven impulse originating in the ionosphere during a brightening of aurora, Planet. Space Sci., 22, 1519-1533, 1974.

Marklund, G. T., Auroral arc classification scheme based on the observed arc-associated electric field pattern, Planet. Space Sci., 31, 193-211, 1984.

Opgenoorth, H. J., I. Haggstrom, P. J. S. Williams, and G. O. L. Jones, Regions of strongly enhanced perpendicular electric fields adjacent to auroral arcs, J. Atmos. Terr. Phys., 52, 449$458,1990$.

Safargaleev, V., W. Lyatsy, and V. Tagirov, Luminosity variations in several parallel auroral arcs before auroral breakup, Ann. Geophysicae, 15, 959-966, 1997.

Timofeev, E. E., M. K. Vallinkoski, T. V. Kozelova, A. G. Yahnin, and R. J. Pellinen, Systematic of arc-associated electric fields and currents as inferred from radar backscatter measurements, J. Geophys., 61, 122-137, 1987.

Williams, P. J. S., R. V. Lewis, T. S. Virdi, M. Lester, and E. Nielsen, Plasma flow bursts in the auroral electrojet, Ann. Geophysicae, 10, 835-848, 1992. 